\title{
Clinical whole-exome sequencing for the diagnosis of rare disorders with congenital anomalies and/or intellectual disability: substantial interest of prospective annual reanalysis
}

\author{
Sophie Nambot, $\mathrm{MD}^{1,2,3,4}$, Julien Thevenon, $\mathrm{MD}, \mathrm{PhD}^{1,3,4}$, Paul Kuentz, MD, $\mathrm{PhD}^{2,3,4}$,
} Yannis Duffourd, $\mathrm{MSc}^{3,4}$, Emilie Tisserant, $\mathrm{PhD}^{3,4}$, Ange-Line Bruel, $\mathrm{PhD}^{3,4}$, Anne-Laure Mosca-Boidron, $\mathrm{MD}^{2,3,4}$, Alice Masurel-Paulet, $\mathrm{MD}^{1,3}$, Daphné Lehalle, $\mathrm{MD}^{1}$, Nolwenn Jean-Marçais, MD ${ }^{1,3}$, Mathilde Lefebvre, $\mathrm{MD}^{1,2}$, Pierre Vabres, $\mathrm{MD}, \mathrm{PhD}^{3,4}$, Salima El Chehadeh-Djebbar, MD ${ }^{1}$, Christophe Philippe, MD, PhD ${ }^{2,4}$, Frederic Tran Mau-Them, MD ${ }^{2,4}$, Judith St-Onge, $\mathrm{DEC}^{2,4}$, Thibaud Jouan, $\mathrm{BSc}^{2,3,4}$, Martin Chevarin, $\mathrm{HNC}^{2,3,4}$, Charlotte Poé, BSc ${ }^{2,3,4}$, Virginie Carmignac, $\mathrm{PhD}^{4}$, Antonio Vitobello, $\mathrm{PhD}^{2,3,4}$, Patrick Callier, $\mathrm{MD}, \mathrm{PhD}^{2,3,4}$, Jean-Baptiste Rivière, $\mathrm{PhD}^{2,3,4}$, Laurence Faivre, $\mathrm{MD}, \mathrm{PhD}^{1,3,4}$, Christel Thauvin-Robinet, $\mathrm{MD}, \mathrm{PhD}^{1,2,3,4}$ and Orphanomix Physicians' Group ${ }^{8}$

Purpose: Congenital anomalies and intellectual disability (CA/ID) are a major diagnostic challenge in medical genetics- $50 \%$ of patients still have no molecular diagnosis after a long and stressful diagnostic "odyssey." Solo clinical whole-exome sequencing (WES) was applied in our genetics center to improve diagnosis in patients with CA/ID.

Methods: This retrospective study examined 416 consecutive tests performed over 3 years to demonstrate the effectiveness of periodically reanalyzing WES data. The raw data from each nonpositive test was reanalyzed at 12 months with the most recent pipeline and in the light of new data in the literature. The results of the reanalysis for patients enrolled in the third year are not yet available.

Results: Of the 416 patients included, data for 156 without a diagnosis were reanalyzed. We obtained 24 (15.4\%) additional diagnoses: 12 through the usual diagnostic process ( 7 new publications, 4 initially misclassified, and 1 copy-number variant), and 12 through translational research by international data sharing. The final yield of positive results was $27.9 \%$ through a strict diagnostic approach, and $2.9 \%$ through an additional research strategy.

Conclusion: This article highlights the effectiveness of periodically combining diagnostic reinterpretation of clinical WES data with translational research involving data sharing for candidate genes.

Genet Med advance online publication 2 November 2017

Key Words: congenital anomalies; data sharing; intellectual disability; reanalysis; whole-exome sequencing

\section{INTRODUCTION}

Congenital anomalies and intellectual disability (CA/ID) comprise a vast, heterogeneous group of disorders, encompassing more than 3,000 different clinical entities, individually rare but collectively frequent. Most CA/ID are of genetic origin and incurred via Mendelian inheritance. Because the prevalence of each disorder is low and a large portion of the molecular bases of CA/ID are still unresolved, their diagnosis remains challenging. These chronic, early-onset disorders contribute significantly to morbidity, mortality, and health- care expenditure, ${ }^{1}$ and their etiologic diagnosis is essential for genetic counseling, prenatal testing, accurate follow-up, prevention of complications, and personalized treatment. ${ }^{2}$ The current standard of care for the diagnosis of CA/ID includes multiple clinical evaluations by specialized physicians, and countless paraclinical investigations such as imaging, metabolic, and biological tests, which are potentially invasive for patients. The genetic investigations include cytogenetic tests and successive single-gene testing, and more recently gene panels. This long and tedious traditional

\footnotetext{
${ }^{1}$ Centre de Génétique et Centre de Référence “Anomalies du Développement et Syndromes Malformatifs,” Hôpital d’Enfants, Centre Hospitalier Universitaire de Dijon, Dijon, France; ${ }^{2}$ UF Innovation en Diagnostic Génomique des Maladies Rares, Centre Hospitalier Universitaire de Dijon, Dijon, France; ${ }^{3}$ Fédération Hospitalo-Universitaire Médecine Translationnelle et Anomalies du Développement, Centre Hospitalier Universitaire de Dijon et Université de Bourgogne-Franche Comté, Dijon, France; ${ }^{4}$ UMR-Inserm 1231 GAD Team, Génétique des Anomalies du Développement, Université de Bourgogne Franche-Comté, Dijon, France. Correspondence: Laurence Faivre or Christel Thauvin-Robinet (laurence.faivre@chu-dijon.fr or christel.thauvin@chu-dijon.fr)

${ }^{8}$ The members of the Orphanomix Group are listed above the References.

The first two authors contributed equally to this work.

The last two authors codirected this work.

Submitted 6 April 2017; accepted 25 July 2017; advance online publication 2 November 2017. doi:10.1038/gim.2017.162
} 
approach leaves approximately half of the families with no diagnosis. ${ }^{3}$

Next-generation sequencing (NGS) has revolutionized medical genetics by improving the chances of obtaining a molecular diagnosis for rare genetic diseases. NGS was initially applied in research, and different strategies were considered to implement NGS for diagnostic purposes. Whole-exome sequencing (WES) has shown an unprecedented success rate in the identification of disease-causing genes in projects ranging from tailored sequencing used to discover the molecular bases of a recognizable syndrome in a homogeneous group of patients, to the systematic application of pan-genomic sequencing in large heterogeneous cohorts. ${ }^{4}$ The usefulness of an unbiased sequencing approach has been highlighted in various heterogeneous disorders, including categories of CA/ID, such as syndromic ID, ${ }^{5}$ developmental delay (DD), ${ }^{6}$ autism, ${ }^{7}$ epilepsy, ${ }^{8}$ and congenital heart defects. ${ }^{9}$

Later, a more accurate interpretation of the data and a reduction in sequencing costs enabled its widespread implementation in clinical practice. Between 2010 and 2015, about 555 genes implicated in Mendelian phenotypes were discovered using NGS. This has resulted in WES becoming the current standard of care for the diagnosis of highly heterogeneous rare disorders with suspected Mendelian inheritance, ${ }^{10}$ thus blurring the line between diagnosis and research. The widespread application of this test in cohorts of patients with undefined CA/ID allows a diagnostic yield ranging from 25 to $32 \%$ (refs. 11-14). This diagnostic yield corresponds to the identification of a disease-causing variant in a gene previously implicated in a human disorder and with a published compatible phenotype. The sequencing strategy may vary from center to center with trio-based or probandbased WES. Although the diagnostic yield should not vary, the likelihood of identifying a candidate variant for a new disorder may depend on the phenotype and the chosen sequencing strategy. ${ }^{13,15}$

This recent acceleration in the discovery of disease-causing genes makes it difficult for physicians to remain up to date with genetic medical knowledge. Initially, the routine use of WES demonstrated the limitations of usual phenotype-driven strategies, based on the clinical expertise of physicians in reference centers for rare diseases, especially in the following situations: (i) atypical presentations of known diseases making it hard to make the diagnosis at first sight; (ii) ultrarare diseases described in very few cases and therefore unknown to most specialists, and (iii) patients exhibiting a specific but only recently discovered phenotype. International data sharing is an efficient solution that overcomes these limitations. By catalyzing the identification of additional patients with similar phenotypic and genotypic profiles, initiatives such as the Matchmaker Exchange project ${ }^{16}$ allow fast and accurate phenotype matching to assess the clinical relevance of candidate variants and genes. Reanalyzing and reinterpreting clinical WES data from large research cohorts is also proving to be an effective way to reveal new diseasecausing variants. In a clinical context, only three articles have assessed the relevance of reanalyzing data. The first focused on data reanalysis and reported an additional diagnostic yield of $10 \%$ in 40 patients. ${ }^{17}$ The second, in a series of 2,000 sequential cases submitted to Ambry Genetics for testing prior to 2016 , showed that $5.6 \%$ of cases that initially received negative or candidate results were upgraded to positive/likely positive or uncertain in a characterized gene. ${ }^{18}$ The third one reported seven changes in the result for 14 reanalyzed cases performed by the molecular laboratory 12 to 18 months after the initial report, of which four resulted in a new definitive diagnosis. ${ }^{19}$ These observations led us to adapt our WESbased clinical practice and diagnostic process by setting up systematic reanalysis and international data sharing.

This retrospective study reports the results and consequences of implementing clinical WES in our current diagnostic practice, and of introducing a systematic reanalysis strategy of unsolved results combined with translational research for candidate genes in a cohort of 416 consecutive patients with CA/ID.

\section{MATERIALS AND METHODS}

\section{Patients}

From June 2013 to June 2016, WES was performed in 323 patients referred to the Reference Center for Congenital Anomalies and Malformative Syndromes in Dijon, France, for an etiological diagnosis and in 93 patients referred to the Orphanomix service (http://www.orphanomix.com/index. html) by other centers in France.

The inclusion criteria were (i) signs of ID or DD when the age of the patient ( $<6$ years) did not permit a diagnosis of ID or the presence of at least one congenital anomaly with or without ID of suspected genetic origin; (ii) a negative prior diagnostic workup; and (iii) informed consent of the patient or parents/guardians for inclusion. Fetuses with multiple malformations were not included in this study. Arraycomparative genomic hybridization was systematically performed before WES in patients with DD, isolated or syndromic ID (associated with dysmorphism or one congenital anomaly), autism spectrum disorders, or pre- or postnatal malformations (two or more), as well as for the characterization of an anomaly detected by another cytogenetic method. In most patients with a convincing diagnostic etiology, a targeted genetic test (single-gene or gene panel) was first ordered. The prescription of WES or gene panel testing was discussed weekly by a group of trained physicians and depended on (i) the clinical and genetic heterogeneity of the suspected disorder and (ii) the availability, turnaround time, and cost of a targeted approach. The conduct of the pretest consultation has been detailed elsewhere. ${ }^{12}$ The local ethics committee approved this study.

\section{Standardized deep phenotyping}

Patients were separated into three major phenotype groups according to the clinical indication: neurodevelopmental disorders, CA without ID/DD, and neuromuscular disorders. The neurodevelopmental disorders group was divided into 

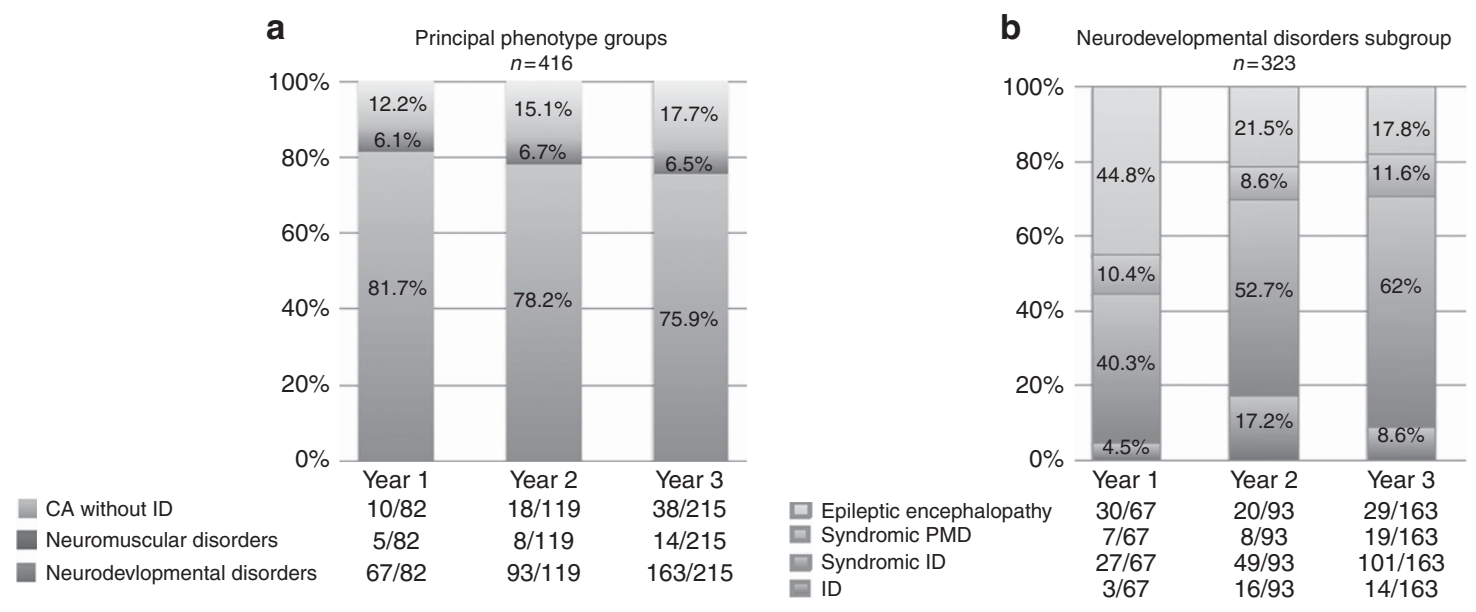

Figure 1 Overview of phenotype distribution in the $\mathbf{4 1 6}$ patients. (a) The phenotype distribution among the 416 patients. (b) The phenotype distribution among the 323 patients of the neurodevelopmental disorders subgroup. CA, congenital anomaly; ID, intellectual disability; PMD, psychomotor delay.

four subgroups: nonsyndromic ID, syndromic ID (defined as ID with CA and/or dysmorphism), epileptic encephalopathy (EE), and syndromic DD (Figure 1b). The proportion of patients with EE was higher during the first year. This initial overrepresentation of patients with $\mathrm{EE}$ can be explained by the work done in our center during the first year of inclusion, which focused on the diagnosis of EE by WES. ${ }^{12}$ Detailed phenotypic data were anonymously collected in the PhenomeCentral database (https://phenomecentral.org/) using the standardized Human Phenotype Ontology terms.

\section{Whole-exome sequencing}

\section{Sequencing and bioinformatics analysis}

In all index cases, libraries of genomic DNA samples were prepared using the Agilent Sureselect Human All Exon v5 kit (Agilent Technologies, Santa Clara, CA), and were sequenced on a HiSeq instrument (Illumina, San Diego, CA) according to the manufacturer's recommendations for paired-end 76-bp reads. The bioinformatics pipeline, alignment processes, and quality procedures have been described elsewhere. ${ }^{12}$ Version 3.4-46 of the Genome Analysis Toolkit was used for this study. Among the 416 patients, 82 (19.7\%) were analyzed during year 1, $119(28.6 \%)$ during year 2, and 215 (51.7\%) during year 3 (Figure 1a).

\section{Copy-number variant detection}

The in-house pipeline for copy-number variant (CNV) detection was developed in November 2015. CNV analysis was retrospectively applied to all patients. The procedure is detailed elsewhere ${ }^{12}$ and in the Supplementary Data online.

\section{Variant interpretation strategy}

The diagnostic interpretation of the filtered variants was done according to the American College of Medical Genetics and Genomics (ACMG) recommendations of 2008 and 2015 (refs. 20,21 ) during the first 2 years and the third year of the study, respectively. The detailed diagnostic interpretation procedure has been reported elsewhere ${ }^{12}$ and is described in the Supplementary Data. The familial segregation study is also detailed in the Supplementary Data. The 56 genes on the list of medically actionable secondary findings defined by the ACMG were also studied and interpreted according to the ACMG recommendations available at the period of reanalysis. ${ }^{22}$ The results were returned to the patient when consent had been given.

\section{Annual reanalysis}

Negative and uncertain results were reanalyzed from the raw sequencing data stored as compressed fastq files (Supplementary Data). All variants of the final analysis file were interpreted. The interpretation first focused on variants previously and newly reported as pathogenic/ probably pathogenic in public databases of clinical interest (ClinVar, http://www.ncbi.nlm.nih.gov/clinvar; DECIPHER, https://decipher.sanger.ac.uk/) or as affecting well-established human disease genes. The interpretation was then extended to all of the other variants, namely those not meeting the diagnostic interpretation criteria. For relevant variants presenting a good genotype-phenotype correlation, but reported in an insufficient number of patients (only one family, one single isolated population) or in several patients of a large cohort without clinical details, we actively searched for additional patients carrying variants in the same gene with a similar phenotype through national collaborations or international data sharing to confirm the genotype-phenotype relationship. This strategy was also used for atypical presentations or new phenotypes linked to an already known gene, but reported only once in the literature or presented in congresses. Reverse phenotyping and data sharing were widely used in these cases to compare and gather patients with the same mutated gene, and look for common clinical features, and thus increase the recurrence. For variants in genes never associated with human disease, the ACMG interpretation criteria were partially applicable. We based on 


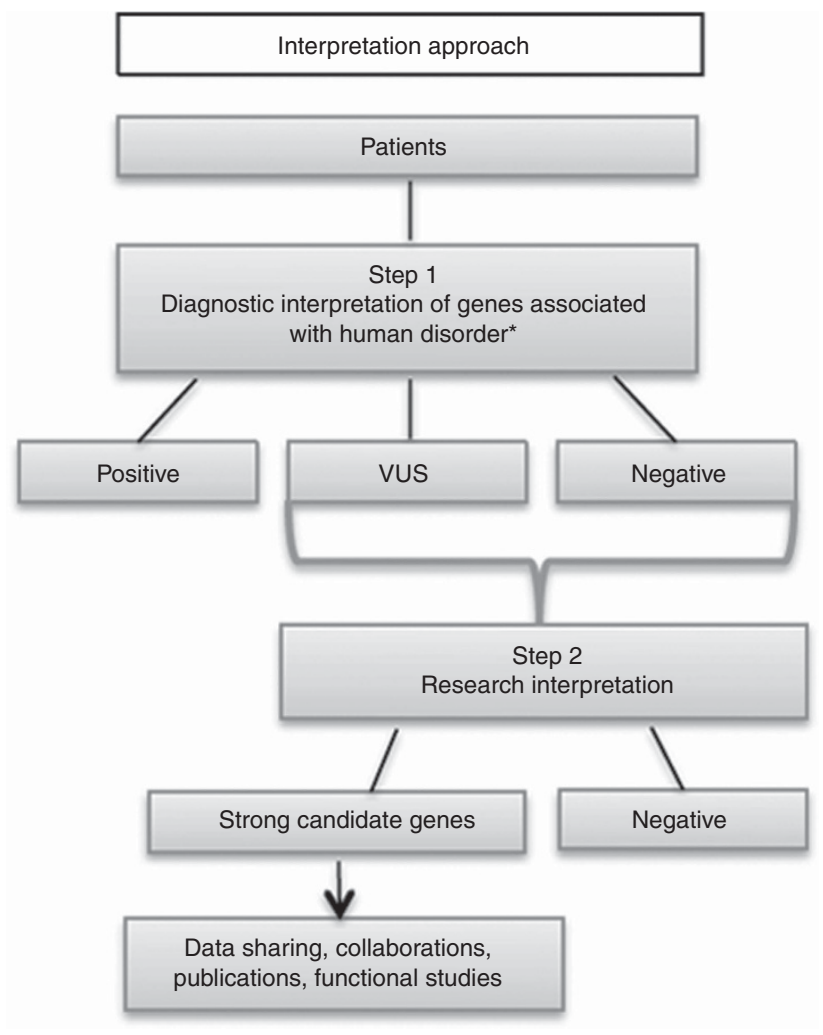

Figure 2 Representation of interpretation approach during reanalysis. *Reported as pathogenic/probably pathogenic in public variant databases of clinical interest (ClinVar) or affecting a gene associated with a human disorder referenced in OMIM (http://www. omim.org) or manually curated via PubMed (https://www.ncbi.nlm.nih. gov/pubmed/); VUS, variant of unknown significance.

the evidence proposed by the ACMG guidelines with particular attention to the encoded protein function, functional studies, animals models, and an intensive search of new patients with a similar phenotype carrying a variant in the same gene through a translational research approach ${ }^{23}$ (Figure 2).

\section{Time to diagnosis}

The time to diagnosis was calculated only for patients seen in our local center with a positive diagnostic result obtained after the first analysis. It corresponded to the overall duration of the diagnostic process, from the first consultation in our center to the date of the WES report.

\section{Patients}

\section{RESULTS}

Between 1 June 2013 and 30 June 2016, 421 patients were referred. Five patients were removed from the analysis because of failed quality control of the sequencing data and the absence of biological samples to repeat the analysis. Overall, 323 patients (77.6\%) were referred by the Reference Center for Congenital Anomalies and Malformative Syndromes of Dijon by seven geneticists, and 93 patients $(22.4 \%)$ were referred by 31 geneticists from 18 French institutions.
The 416 patients included 171 females (41\%) and 245 males $(59 \%)($ sex ratio $=1.4)$, with $84.4 \%$ children (351 patients) and $15.6 \%$ adults (65 patients). The mean age of patients when samples were sent for sequencing was 6.7 years for children and 30.7 for adults (global average: 10.5 years). Thirty-seven patients were born to consanguineous parents.

\section{Molecular results}

Over the 3 years and after two reanalyses, 128 of the initial 416 patients (30.8\%) received a positive molecular diagnosis. The distribution of the Mendelian mode of inheritance and variant type are presented in Figure 3. Of the 79 autosomaldominant conditions diagnosed, $64(81 \%)$ arose as a result of a de novo variant, 11 (13\%) were inherited, and four (5\%) were undetermined due to a lack of parental samples. Among the 34 autosomal-recessive disorders, there were $16(45.7 \%)$ cases of homozygosity, and 18 (51.4\%) cases of compound heterozygosity. Of the $15 \mathrm{X}$-linked disorders, 8 (53\%) were linked to a de novo variant and $7(47 \%)$ were inherited. The three pathogenic or probably pathogenic CNVs included only deletions, which ranged from 0.637 to $4.5 \mathrm{~kb}$ (Supplementary Table S1a). The resolution of the array-comparative genomic hybridization was not sufficient to detect these CNVs. The 148 pathogenic or likely pathogenic single-nucleotide variants or CNVs occurred in 104 different genes.

In addition, 39/416 patients (9.4\%) remained with an uncertain result (Figure 3).

\section{Molecular results after the initial analysis}

The global initial diagnostic yield was 25\% (104/416), ranging from $22 \%$ in year 1 to $27.4 \%$ in year 3 (Figure 4). Six of the 416 patients had a medically actionable secondary finding in one of the 56 ACMG genes (Supplementary Table S1b) and 2 of these 6 patients harbored a secondary finding with no etiological diagnosis. The pathogenic and probably pathogenic variants, corresponding to a positive result, and the variants of secondary findings are being submitted to the ClinVar database (submission ID: SUB2609258).

\section{Annual reanalysis}

The first annual reanalysis was performed for patients with a nonpositive result included in year 1 (64/82 patients) and in year 2 (92/119 patients), meaning 156 of the 416 (37.5\%) patients. In the first reanalysis for year 1 patients, we obtained one additional positive result (1.2\%) using a strict diagnostic approach and four (4.8\%) by a translational research approach, leading to an additional diagnostic yield of $6 \%$ $(5 / 82)$. For the first reanalysis for year 2 patients, we obtained 10 additional diagnoses by a diagnostic approach, corresponding to a supplemental diagnostic yield of $8 \%(10 / 119)$ and $2(1.7 \%)$ by a translational research approach. In the second reanalysis for year 1 patients, we obtained one additional positive result (1.2\%) using a strict diagnostic approach and six $(7.3 \%)$ by a translational research approach (Figure 4). The results for the reanalysis of patients included in year 3 are not complete and not yet available because the 

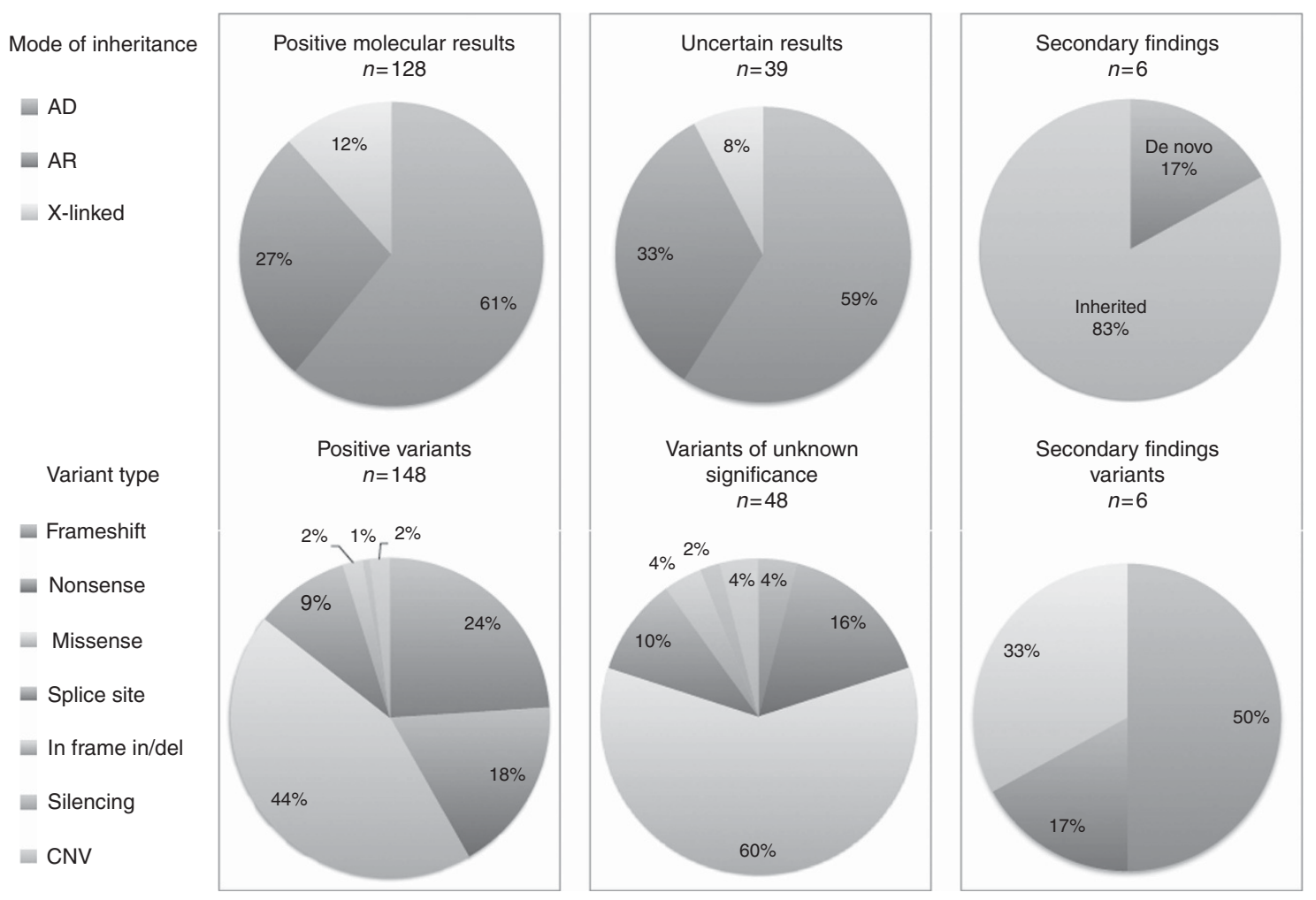

Figure 3 Distribution of Mendelian mode of inheritance and variant type for cases with positive molecular diagnosis, variants of unknown significance, and secondary findings. $A D$, autosomal dominant; $A R$, autosomal recessive; CNV, copy-number variant.

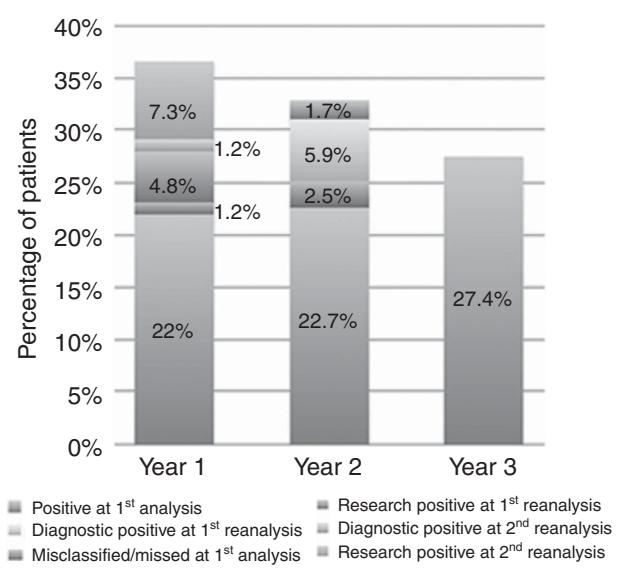

Figure 4 Evolution of diagnostic yield regarding prospective reanalysis.

period of 12 months was not finished at the time of submission.

Finally, among the 156 reanalyzed patients, 24 results (15\%) became positive. Of these 24 positive results, 14 were initially negative, and 10 were initially uncertain because of the lack of data in the literature or because of the nondetection of a second event in recessive disorders. Twelve of the 24 cases were reassessed through usual diagnostic processes thanks to a recent publication, reconsideration of the first interpretation, or the detection of a CNV (Table 1). The other 12 cases were resolved through a translational research strategy using proactive international data sharing and publications of our team or in collaboration with other teams. This strategy allowed us to identify at least five novel genes, not previously associated with a human disease $\left(K C N A 2,{ }^{24} \mathrm{FIBP},{ }^{25} \mathrm{AP} 3 \mathrm{~B} 2,{ }^{26}\right.$ SLC13A5 ${ }^{27}$ and $\left.M A B 21 L 1^{28}\right)$. We collected as much evidence as possible to fulfill the ACMG criteria and thus to consider these results as certain/diagnostic results.

\section{Phenotype distribution and diagnostic yield per phenotype group}

Over the 3 years, we observed a globally similar distribution of the phenotype groups with a majority of patients presenting neurodevelopmental disorders $(n=323)$, which is in keeping with the recruitment criteria for our cohort (Figure 1a).

The highest global diagnostic yield after two reanalysis procedures was obtained for the neuromuscular disorders group (33\%), followed by the neurodevelopmental disorders group (31.5\%) (Supplementary Figure S1). Chi-squared tests did not show any significant difference in the diagnostic yield between groups. We observed an increase in positive results at each reanalysis for neurodevelopmental disorders, and a clear improvement for syndromic ID and EE (Supplementary Figure S2).

\section{Time to diagnosis}

Over the 3 years, the median time to diagnosis was reduced by 9 months (Supplementary Figure S3). The reduction was 
Table 1 Diagnoses obtained by reanalysis of WES data

$\begin{array}{lll}\begin{array}{l}\text { Cause of } \\ \text { change }\end{array} & \begin{array}{l}\text { Diagnosis from reanalysis } \\ \text { of WES data }\end{array} & \begin{array}{l}\text { Date of first } \\ \text { WES analysis }\end{array}\end{array}$

Usual diagnostic approach: publication date of article of interest

Translational research approach: publication date of article of interest

Recent publication

Claes-Jensen type syndromic

January 2015

January 2012 and February 2015

X-linked mental retardation

(MIM 300534)

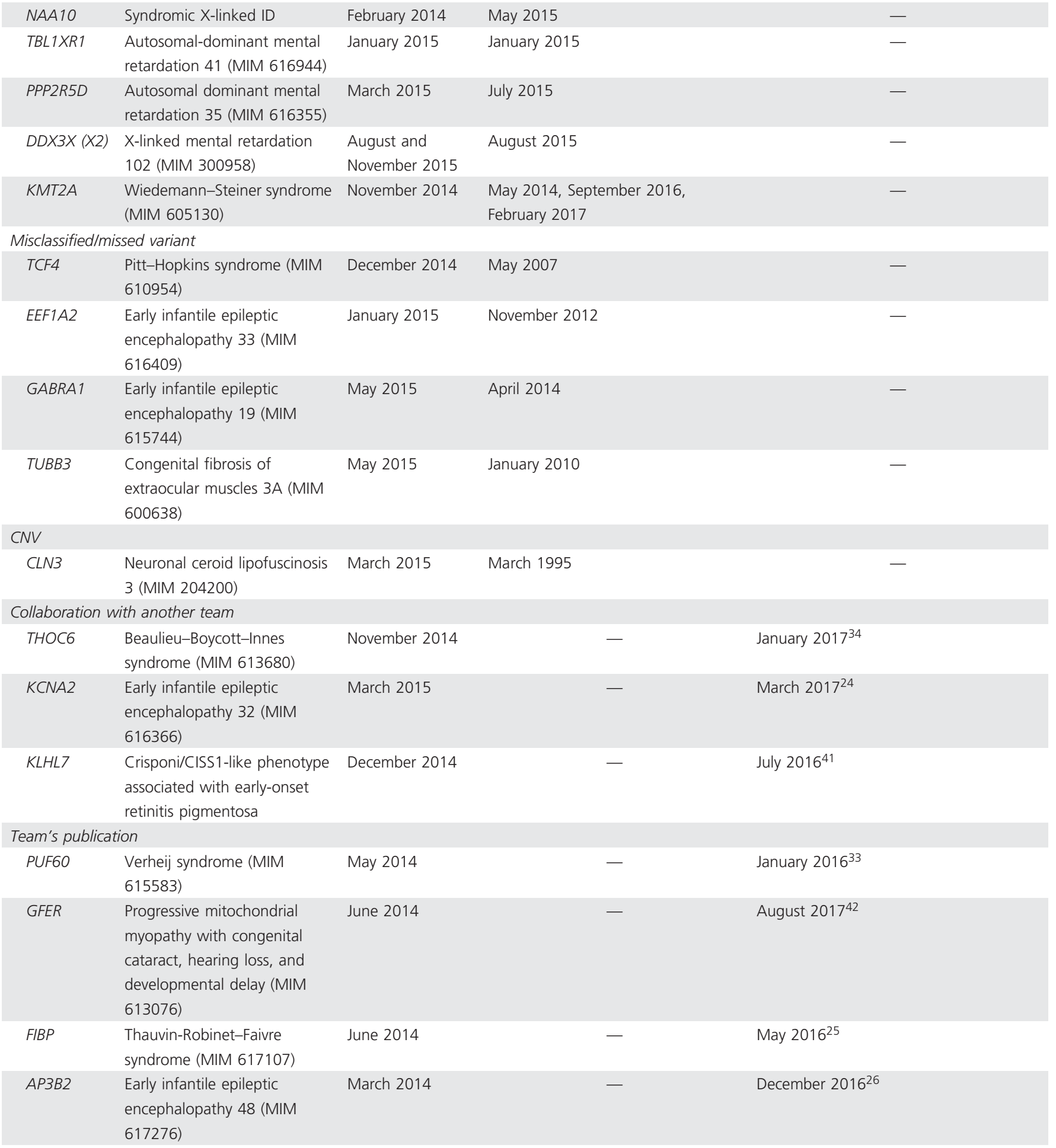


Table 1 Continued

\begin{tabular}{|c|c|c|c|c|}
\hline $\begin{array}{l}\text { Cause of } \\
\text { change }\end{array}$ & $\begin{array}{l}\text { Diagnosis from reanalysis } \\
\text { of WES data }\end{array}$ & $\begin{array}{l}\text { Date of first } \\
\text { WES analysis }\end{array}$ & $\begin{array}{l}\text { Usual diagnostic approach: } \\
\text { publication date of article of } \\
\text { interest }\end{array}$ & $\begin{array}{l}\text { Translational research approach: } \\
\text { publication date of article of interest }\end{array}$ \\
\hline $\begin{array}{l}\text { SLC13A5 } \\
(\times 2)\end{array}$ & $\begin{array}{l}\text { Early infantile epileptic } \\
\text { encephalopathy } 25 \text { (MIM } \\
615905 \text { ) }\end{array}$ & November 2013 & - & July $2014^{27}$ \\
\hline$M A B 21 L 1$ & Syndromic scrotal agenesis & November 2014 & - & February $2017^{28}$ \\
\hline PACS2 & Syndromic ID & May 2015 & - & \\
\hline BRWD3 & $\begin{array}{l}\text { X-linked mental retardation } 93 \\
\text { (MIM 300659) }\end{array}$ & May 2015 & - & \\
\hline
\end{tabular}

CISS, cold-induced sweating syndrome; ID, intellectual disability; WES, whole-exome sequencing.

greatest between the first and the second year (6 months), which is in keeping with the complete implementation of diagnostic WES in our current diagnostic process in June 2014.

Time devoted to research reanalysis by the laboratory and clinical teams

It took the bioinformaticians a few hours to update the inhouse pipeline and reanalyze the raw data, but pipeline development for $\mathrm{CNV}$ detection required approximately 600 hours of work. After negative diagnostic result, it took a trained interpreter on average three hours to analyze variants via a research process and a variable amount of time for multidisciplinary discussions. Supplementary Sanger validations should also be considered (one or two per patient; probably excessive because of the solo strategy), requiring additional time for technicians and other techniques. The physicians provided accurate, updated phenotype data by regular consultations, by asking patients and their family to return for reverse phenotyping or to extend the familial segregation, by answering numerous mails in case of collaborations, and by collecting data for recruited patients through data sharing. Time was also spent explaining new results to patients. The time devoted to each patient varied widely, ranging from a few hours to a few days. Finally, the report update took an average of 30 minutes.

\section{DISCUSSION}

This retrospective study reports the molecular results of proband-based clinical WES in 416 consecutive patients with CA/ID included over 3 years. It highlights the increase in diagnostic yield thanks to the yearly reanalysis and interpretation of raw WES data. We obtained a global diagnostic yield of $27.9 \%$ after two reanalyses, and 12 (2.9\%) more cases were resolved through the combined diagnostic/research approach.

Considering the exponential evolution of genetic discoveries and the improvements in computer performance over the past 5 years, prospective reanalysis of nonpositive WES results appears manageable and worthwhile for undiagnosed patients. This strategy allowed us to obtain 24 (15\%) additional positive diagnoses among the 156 nonpositive initial results. Recent publications allowed us to assign a positive result after reanalysis in five initially negative cases and two uncertain cases (4.5\%). This principally concerned patients included in the second year and is consistent with other studies, ${ }^{17-19}$ one of which reported that $73 \%(70 / 96)$ of reclassifications were linked to the discovery of new genedisease relationships described in the medical literature. ${ }^{18}$ It also allowed us to reclassify uncertain missense variants occurring in a human disease gene, in which only truncating mutations were reported as pathogenic in the first publications. Wiedemann-Steiner syndrome due to KMT2A variants, now with three published pathogenic missense variants, is a good example of this situation. ${ }^{29}$ In addition to this effective way to resolve unsolved cases, some variants became positive after reanalysis because of an initial misinterpretation, allowing four additional diagnoses (2.5\%). This phenomenon has been underscored in several studies reporting the difficulty of determining the pathogenicity of a variant, considering the extremely heterogeneous evidence taken into account. ${ }^{18,30-32}$ In 2016, Amendola et al. ${ }^{30}$ reported a rate of variant discordance between nine laboratories of $66 \%$ for 99 analyzed variants, despite the application of the ACMGAssociation for Molecular Pathology guidelines. Among the differences found, $22 \%$ might affect medical management. One additional diagnosis (1\%) was made possible thanks to the upgrade of the pipeline for detecting CNVs. Finally, a search of published data in the context of diagnostic reanalysis led to a positive diagnosis in $12 / 156$ cases $(7.7 \%)$, which is similar to recently published data in a large number of patients. ${ }^{17}$

The major reason for our high number of positive results after reanalysis was the active search for additional evidence of pathogenicity for strong candidate variants, leading to 12 additional positive diagnoses $(7.7 \%)$, with nine publications by our team or collaborations with another team, including seven through the Matchmaker Exchange initiative $24-27,33,34$ (Table 1). This tool has become essential to rapidly identify new patients carrying a variant in the same gene, thus enhancing the identification of the molecular basis of ultrarare disorders of suspected Mendelian inheritance. This integrated translational strategy blurs the line between a standardized diagnostic procedure and a dedicated research procedure, involving a research team for this task. ${ }^{18,35}$ 
These results were directly linked to a complementary strategy that combined usual diagnosis techniques with translational research and showed the importance of (i) tools to aid variant classification such as ClinGen; ${ }^{36}$ (ii) double interpretation and regular multidisciplinary discussions in the laboratory, and also the sharing of practices between laboratories; (iii) data sharing for the available genomic data of affected patients, asymptomatic control individuals, and different ethnic populations; (iv) need for training; and (v) regular reanalysis.

For the $30.7 \%$ of patients given a positive molecular result, this step not only ended a diagnostic "odyssey," but also provided precious and accurate information for medical care for patients and their families. Novel therapeutic options or recommendations for clinical care were introduced following positive WES diagnosis in nine patients. For example, a cetogenic diet was started in a girl carrying a variation in SLC2A1 (GLUT1 deficiency syndrome 1 (MIM 606777)). This diet stopped the seizures and clearly improved the psychomotor development.

In the remaining nonpositive cases, prospective WES reanalysis should be continued because the causal variant(s) is probably located in genes not yet known to be associated with disease, ${ }^{10}$ or because the phenotype is characterized by multiple molecular diagnoses. In our cohort, we identified only one patient with a double molecular diagnosis, which included a homozygous variant in HPS3 associated with Hermansky-Pudlak syndrome, and a heterozygous variant in TTPA, explaining the ataxia present in his brother, who carried this variant in a homozygous state. Therefore, multiple diagnoses are probably underidentified and physicians must keep in mind that the entire phenotype is not necessarily explained by only one diagnosis. Indeed, our results were under the $5 \%$ found by Posey et al. ${ }^{37}$ in their retrospective study, which included 7,374 patients who underwent proband or trio-based diagnostic WES. Interpreters and physicians must be vigilant and make a critical appraisal of positive results, particularly for syndromes reported only once or twice.

This study highlighted that clinical WES, together with the regular reanalysis of nonpositive results, is a powerful tool in the diagnosis of CA/ID. Its early use in the diagnostic strategy reduces the time to diagnosis. This reduction is also explained by the disruption of our diagnostic reasoning, which was initially based on a phenotype-driven approach. In the case of ultrarare or atypical phenotypes, which are frequent in syndromic ID, reverse phenotyping is an important tool to help in the interpretation of WES results. It involves an accurate reexamination of the clinical data, to compare them with phenotypes described in the literature. ${ }^{38}$ The use of this genotype-driven approach, as a complement to the traditional phenotype-driven approach, allows the more efficient ordering of complementary exams, which will probably lead to a cost reduction. This has been illustrated by several medicoeconomic studies in subjects with a suspected genetic condition. All of these studies highlighted the ability of
WES to establish a molecular diagnosis at a lower cost than is the case with classical techniques. ${ }^{39,40}$

Although sequencing did not lead to additional costs, the organization of systematic reanalysis led to a sizeable workload at different steps of the process. First, bioinformatics updates and the reanalysis of raw data are quite fast, but because the software is constantly being updated, regular improvements of the pipeline are required to maximize technological performance. Second, there is a considerable increase in technical procedures that need to be automated. Finally, participation of clinicians is required for reverse phenotyping and data sharing, which are more and more frequently used in the research approach. This annual reanalysis strategy is a great advantage for patients, but seems difficult to integrate into the current activity of a clinical laboratory without major adaptations. Indeed, research reanalysis is time-consuming (on average three hours per patient), and could be systematized only if a sufficiently large research team works in partnership with the clinical laboratory.

In conclusion, our study underscores the considerable interest of prospective reanalysis of WES data in patients with no diagnosis. For atypical and ultrarare disorders, the border between diagnosis and research is becoming increasingly thin, thus underlining the need for a translational integrated organization from diagnosis to research to provide careful and reliable interpretation of data. For the remaining negative results of solo WES despite recurrent reanalysis, a trio strategy, to identify de novo candidate variants, could be the first step before the prescription of whole-genome sequencing and other omics technologies to determine molecular etiologies that remain undetectable by WES. When the cost of whole-genome sequencing decreases, the whole-genome sequencing-first approach will be the best option to tend toward a unique diagnostic test.

\section{SUPPLEMENTARY MATERIAL}

Supplementary material is linked to the online version of the paper at http://www.nature.com/gim

\section{ACKNOWLEDGMENTS}

We thank the Regional Council of Burgundy and the Centre Hospitalo-Universitaire de Dijon for financial support. We also thank the University of Burgundy Centre de Calcul for technical support and management of the informatics platform, and the Biomedicine Agency for structural variation software XHMM under the call for tender "AMP, diagnostic prénatal et diagnostic génétique." Finally, we thank the patients and their families involved in the study, and the physicians who referred their patients to our center.

\section{AUTHOR'S AFFILIATIONS OF THE ORPHANOMIX GROUP}

Jean-Luc Alessandri (Pôle Enfants, Centre Hospitalier Universitaire de la Réunion, Saint-Denis, France); Yline Capri (Unité de Génétique Clinique, Département de Génétique, Centre Hospitalier Universitaire de Paris-Hôpital Robert Debré, Paris, France); Marie-Pierre Cordier-Alex (Service de Génétique Clinique, 
Hospices Civils de Lyon, Bron, France); Christine Coubes (Département de Génétique Médicale, Centre Hospitalier Régional Universitaire de Montpellier, Montpellier, France); François Feillet (Service de Médecine Infantile, Centre Hospitalier Universitaire de Nancy, Nancy, France); Mélanie Fradin (Service de Génétique Clinique, Centre Hospitalier Universitaire de Rennes, Rennes, France); David Geneviève (Département de Génétique Médicale, Centre Hospitalier Régional Universitaire de Montpellier, Montpellier, France); Marion Gérard-Blanluet (Service de Génétique, Centre Hospitalier Universitaire de Caen, Caen, France); Dominique Germain (Service de Génétique Médicale, Hôpital Raymond Poincaré, Paris, France); Brigitte GilbertDussardier (Service de Génétique Médicale, Centre Hospitalier Universitaire de Poitiers, Poitiers, France); Cyril Goizet (Service de Génétique Médicale, Centre Hospitalier Universitaire de Bordeaux-GH Pellegrin, Bordeaux, France); Alice Goldenberg (Service de Génétique, Centre Hospitalier Universitaire de Rouen, Rouen, France); Delphine Heron (Service de Génétique Clinique, Unité Fonctionnelle de Génétique Médicale, Centre Hospitalier Universitaire de Paris-GH La Pitié Salpêtrière-Charles Foix, Paris, France); Marie-Line Jacquemont (Unité de Génétique Médicale, Centre Hospitalier Universitaire de La Réunion, Site GHSR, France); Aurelia Jacquette (Service de Génétique Clinique, Unité Fonctionnelle de Génétique Médicale, Centre Hospitalier Universitaire de Paris-GH La Pitié Salpêtrière-Charles Foix, Paris, France); Elodie Lacaze (Service de Génétique, Centre Hospitalier Universitaire de Caen, Caen, France); Didier Lacombe (Service de Génétique Médicale, Centre Hospitalier Universitaire de Bordeaux-GH Pellegrin, Bordeaux, France); Fanny Laffargue (Service de Génétique Médicale, Centre Hospitalier Universitaire de Clermont-Ferrand, Clermont-Ferrand, France); Laëtitia Lambert (Service de Médecine Infantile et Génétique Clinique, Centre Hospitalier Universitaire de Nancy, Nancy, France); Béatrice Laudier (Structure Interne de Génétique-INEM-UMR7355CNRS, Centre Hospitalier Régional d'Orléans, Orléans, France); Marine Lebrun (Service de Génétique Médicale, Centre Hospitalier Universitaire de Saint-Etienne, Saint-Priez-en-Jarez, France); Isabelle Maystadt (Centre de Génétique Humaine, Institut de Pathologie et Génétique, Gosselies (Charleroi), Belgium); Fanny Morice-Picard (Service de Génétique Médicale, Centre Hospitalier Universitaire de Bordeaux-GH Pellegrin, Bordeaux, France); Sébastien Moutton (Laboratoire Maladies Rares: Génétique et Métabolisme, Centre Hospitalier Universitaire de Bordeaux-GH Pellegrin, Bordeaux, France); Laurence Perrin (Unité de Génétique Clinique, Centre Hospitalier Universitaire de Paris-Hôpital Robert Debré, Paris, France); Florence Petit (Service de Génétique Clinique, Centre Hospitalier Régional Universitaire de Lille, Lille, France); Fabienne Prieur (Service de Génétique Clinique Chromosomique et Moléculaire, Centre Hospitalier Universitaire de SaintEtienne, Saint-Priez-en-Jarez, France); Pierre Sarda (Département de Génétique Médicale, Centre Hospitalier Régional Universitaire de Montpellier, Montpellier, France); Elise Schaefer (Service de Génétique Médicale, Institut de Génétique Médicale d'Alsace, Hôpitaux Universitaires de Strasbourg, Strasbourg, France); Renaud Touraine (Service de Génétique Médicale, Centre Hospitalier Universitaire de Saint-Etienne, Saint-Priez-en-Jarez,
France); Alain Verloes (Département de Génétique, Hôpital Robert Debré, Paris, France); Marjolaine Willems (Département de Génétique Médicale, Centre Hospitalier Régional Universitaire de Montpellier, Montpellier, France).

\section{DISCLOSURE}

The authors declare no conflict of interest.

\section{REFERENCES}

1. McCandless SE, Brunger JW, Cassidy SB. The burden of genetic disease on inpatient care in a children's hospital. Am J Hum Genet 2004;74: 121-127.

2. Schieppati A, Henter J-I, Daina E, Aperia A. Why rare diseases are an important medical and social issue. Lancet 2008;371:2039-2041.

3. Shashi V, McConkie-Rosell A, Rosell B, et al. The utility of the traditional medical genetics diagnostic evaluation in the context of next-generation sequencing for undiagnosed genetic disorders. Genet Med 2014;16: 176-182.

4. $\mathrm{Ng} \mathrm{SB}$, Buckingham $\mathrm{KJ}$, Lee $\mathrm{C}$, et al. Exome sequencing identifies the cause of a Mendelian disorder. Nat Genet 2010;42:30-35.

5. Vissers LE, Gilissen C, Veltman JA. Genetic studies in intellectual disability and related disorders. Nat Rev Genet 2016;17:9-18.

6. Deciphering Developmental Disorders Study. Large-scale discovery of novel genetic causes of developmental disorders. Nature 2015;519: 223-228.

7. Iossifov I, O'Roak BJ, Sanders SJ, et al. The contribution of de novo coding mutations to autism spectrum disorder. Nature 2014;515:216-221.

8. Helbig KL, Farwell Hagman KD, Shinde DN, et al. Diagnostic exome sequencing provides a molecular diagnosis for a significant proportion of patients with epilepsy. Genet Med 2016;18:898-905.

9. Long PA, Larsen BT, Evans JM, Olson TM. Exome sequencing identifies pathogenic and modifier mutations in a child with sporadic dilated cardiomyopathy. J Am Heart Assoc 2015;4:e002443.

10. Chong JX, Buckingham KJ, Jhangiani SN, et al. The genetic basis of Mendelian phenotypes: discoveries, challenges, and opportunities. Am J Hum Genet 2015;97:199-215.

11. Lee $H$, Deignan JL, Dorrani $N$, et al. Clinical exome sequencing for genetic identification of rare Mendelian disorders. JAMA 2014;312:1880-1887.

12. Thevenon J, Duffourd $Y$, Masurel-Paulet A, et al. Diagnostic odyssey in severe neurodevelopmental disorders: toward clinical whole-exome sequencing as a first-line diagnostic test. Clin Genet 2016;89:700-707.

13. Yang $Y$, Muzny DM, Xia F, et al. Molecular findings among patients referred for clinical whole-exome sequencing. JAMA 2014;312: 1870-1879.

14. Iglesias A, Anyane-Yeboa K, Wynn J, et al. The usefulness of wholeexome sequencing in routine clinical practice. Genet Med 2014;16: 922-931.

15. Wright CF, Fitzgerald TW, Jones WD, et al. Genetic diagnosis of developmental disorders in the DDD study: a scalable analysis of genomewide research data. Lancet 2015;385:1305-1314.

16. Philippakis AA, Azzariti DR, Beltran S, et al. The Matchmaker Exchange: a platform for rare disease gene discovery. Hum Mutat 2015;36:915-921.

17. Wenger AM, Guturu H, Bernstein JA, Bejerano G. Systematic reanalysis of clinical exome data yields additional diagnoses: implications for providers. Genet Med 2017;19:209-214.

18. Smith ED, Radtke K, Rossi M, et al. Classification of genes: standardized clinical validity assessment of gene-disease associations aids diagnostic exome analysis and reclassifications. Hum Mutat 2017;38:600-608.

19. Baldridge D, Heeley J, Vineyard M, et al. The Exome Clinic and the role of medical genetics expertise in the interpretation of exome sequencing results. Genet Med. 2017;19:1040-1048.

20. Richards CS, Bale S, Bellissimo DB, et al. ACMG recommendations for standards for interpretation and reporting of sequence variations: revisions 2007. Genet Med 2008;10:294-300.

21. Richards S, the ACMG Laboratory Quality Assurance Committee. Standards and guidelines for the interpretation of sequence variants: a joint consensus recommendation of the American College of Medical Genetics and Genomics and the Association for Molecular Pathology. Genet Med. 2015;17:405-424. 
22. Green RC, Berg JS, Grody WW, et al. ACMG recommendations for reporting of incidental findings in clinical exome and genome sequencing. Genet Med 2013;15:565-574.

23. MacArthur DG, Manolio TA, Dimmock DP. Guidelines for investigating causality of sequence variants in human disease. Nature 2014;508: 469-476.

24. Allou L, Julia S, Amsallem D, et al. Rett-like phenotypes: expanding the genetic heterogeneity to the KCNA2 gene and first familial case of CDKL5-related disease. Clin Genet 2017;91:431-440.

25. Thauvin-Robinet C, Duplomb-Jego L, Limoge F, et al. Homozygous FIBP nonsense variant responsible of syndromic overgrowth, with overgrowth, macrocephaly, retinal coloboma and learning disabilities. Clin Genet 2016;89:e1-4

26. Assoum M, Philippe C, Isidor B, et al. Autosomal-recessive mutations in AP3B2, adaptor-related protein complex 3 beta 2 subunit, cause an earlyonset epileptic encephalopathy with optic atrophy. Am J Hum Genet 2016;99:1368-1376.

27. Thevenon J, Milh M, Feillet F, et al. Mutations in SLC13A5 cause autosomal-recessive epileptic encephalopathy with seizure onset in the first days of life. Am J Hum Genet 2014;95:113-120.

28. Bruel AL, Masurel-Paulet A, Rivière JB, et al. Autosomal recessive truncating MAB21L1 mutation associated with a syndromic scrotal agenesis. Clin Genet 2017;91:333-338.

29. Min Ko J, Cho JS, Yoo Y, et al. Wiedemann-Steiner syndrome with 2 novel KMT2A mutations. J Child Neurol. 2017;32:237-242.

30. Amendola LM, Jarvik GP, Leo MC, et al. Performance of ACMG-AMP variantinterpretation guidelines among nine laboratories in the Clinical Sequencing Exploratory Research Consortium. Am J Hum Genet 2016;99:247.

31. O'Daniel JM, McLaughlin HM, Amendola LM, et al. A survey of current practices for genomic sequencing test interpretation and reporting processes in US laboratories. Genet Med 2016;19:575-582.

32. Manrai AK, Funke BH, Rehm HL, et al. Genetic misdiagnoses and the potential for health disparities. N Engl J Med 2016;375:655-665.
33. El Chehadeh S, Kerstjens-Frederikse WS, Thevenon J, et al. Dominant variants in the splicing factor PUF60 cause a recognizable syndrome with intellectual disability, heart defects and short stature. Eur J Hum Genet 2016;25:43-51

34. Amos JS, Huang $L$, Thevenon J, et al. Autosomal recessive mutations in THOC6 cause intellectual disability: syndrome delineation requiring forward and reverse phenotyping. Clin Genet 2017;91:92-99

35. Bowdin S, Gilbert A, Bedoukian $E$, et al. Recommendations for the integration of genomics into clinical practice. Genet Med. 2016;11: 1075-1084.

36. Rehm HL, Berg JS, Brooks LD, et al. ClinGen-the clinical genome resource. N Engl J Med 2015;372:2235-2242.

37. Posey JE, Harel T, Liu P, et al. Resolution of disease phenotypes resulting from multilocus genomic variation. N Engl J Med 2017;376:21-31.

38. Nambot S, Masurel A, El Chehadeh S, et al. 9q33.3q34.11 Microdeletion: new contiguous gene syndrome encompassing STXBP1, LMX1B and ENG genes assessed using reverse phenotyping. Eur J Hum Genet 2016;24: 830-837.

39. Stark Z, Schofield D, Alam K, et al. Prospective comparison of the costeffectiveness of clinical whole-exome sequencing with that of usual care overwhelmingly supports early use and reimbursement. Genet Med 2017;19:867-874.

40. Valencia CA, Husami A, Holle J, et al. Clinical impact and costeffectiveness of whole exome sequencing as a diagnostic tool: a pediatric center's experience. Front Pediatr 2015;3:67.

41. Angius A, Uva P, Buers I, et al. Bi-allelic Mutations in KLHL7 Cause a Crisponi/CISS1-like Phenotype Associated with Early-Onset Retinitis Pigmentosa. Am J Hum Genet. 2016;99:236-245.

42. Nambot S, Gavrilov D, Thevenon J, et al. Further delineation of a rare recessive encephalomyopathy linked to mutations in GFER thanks to data sharing of whole exome sequencing data. Clin Genet. 2017;92: 188-198. 